\title{
DEFLECTIONS AND FREQUENCY ANALYSIS IN THE MILLING OF THIN-WALLED PARTS WITH VARIABLE LOW STIFFNESS
}

\author{
Serhit Kononenko, Sergey Dobrotvorskiy, Yevhenita Basova*, \\ Magomediemin Gasanov, Ludmila Dobrovolska
}

\author{
National Technical University "Kharkiv Polytechnic Institute", Kyrpychova str. 2, Kharkiv, Ukraine \\ * corresponding author: e.v.basova.khpi@gmail.com
}

\begin{abstract}
The disadvantage of the geometry of thin-walled parts, in terms of processing, is the low ability to resist static and dynamic loads. It is caused by the elastic deformation of elements with a low stiffness. Modelling approaches for the evaluation of deflections during machining are presented. Mathematical models of deflections, cutting forces and harmonic response are proposed. The processes of material removal and deflection of a thin-walled sample at the critical points are modelled. A frequency analysis was performed, consisting of a modal analysis of natural frequencies and a harmonic response analysis. As a result, a graph of the deflections amplitude from the frequency of driven harmonic oscillations is generated. The analysis of the obtained values was performed. As a result, the resonance frequency and maximum amplitude of oscillations for the operating parameters are determined.
\end{abstract}

KEYworDs: Thin-walled parts, undesirable deflections, end milling, modal analysis, harmonic response analysis.

\section{INTRODUCTION}

In several branches of the industry, such as mechanical engineering, aircraft manufacturing, drive production, etc., engineering products that belong to a number of thin-walled parts with variable stiffness are particularly prevalent. The processing of such parts on metal-cutting machines generally requires the application of both the specially developed devices that prevent the parts from being deformed by cutting forces and fixing or using new mechanical treatment techniques. The creation and using of special devices is associated with additional costs and, as a result, with an increase in the cost of production. The transition to an adoption of contemporary mechanical treatment methods such as high-speed milling reduces the number of special technological equipment. However, this mechanical treatment technique needs to be studied as well and the positive impact of reduced cutting forces influence on the thing-walled element should be approved. The aim of the research is to develop the technological solutions to reduce undesirable deviations of the geometry of thin-walled elements of parts by high-speed end milling.

\section{LiterATURE REVIEW}

Parts, such as turbine blades, impellers, and similar that have thin-walled elements in their geometry, are indispensable in the automobile, aerospace and other industries [1-4]. Such responsible parts of the mechanisms are used in a variety of drive units [5].

The formation of surfaces of thin-walled parts with variable low stiffness requires a consideration of a number of factors that could prevent the achievement of technological requirements for the product [6, 7]. Such factors are: deviations from a given shape that increases during processing, vibrations, thermal deformations and errors caused by tools, tool-path accuracy, technological equipment [8, 9] and fixtures [10, 11.

In modern industry and research activity, methods for recording and analysing the parameters of the cutting process for thin-walled parts are being actively developed. One of the methods based on a prediction of cutting parameters is discrete-time modelling of dynamic milling systems. The discrete-time model is general, and it can be used simultaneously for predicting the stability as well as the time response of the milling system [12].

An experimental based study provides a systematic measurement of process response parameters, videlicet the cutting force and surface roughness [13, dynamic stiffness measuring methods [14]. In addition, a special attention is paid to the system identification, signal, modal analysis, vibration absorption [15-18] using the frame structures as a test rig for numerical identification techniques.

Another researches represent a methods based on a variable correction designed to compensate the deflection effect of a non-rigid construction of blades [19] and technological and other factors of the accuracy characteristics of the blades processing. The correction values depend on the contact area of the tool with the workpiece. The converted parametric view of the machining program makes it possible to include compensating values to the machine manually by the operator. In the process, the operator checks the correctness of the processing. In the case of incorrect 
values of correction, the program should be changed and processing starts again [20].

A research based on a simulation of the distortion due to machining of thin-walled components states that the distortion of components is strongly related to the residual stress state induced by manufacturing processes like heat treatment, forming or machining [21].

As a result of the review, it was decided to focus on the forces in the process of the material removal, FE end-milling modelling and oscillations analysis to determine the undesirable deflections degree. For basic estimation and further validation, a simplified model of a part is considered as a cantilever beam [22, 23] that corresponds to an earlier study [24] where it was clarified that the maximum of deviation occurs on the ending free point of the sample.

\section{ReSEARCH METhodology}

The technological solution for determining the values of undesirable deflections, in this paper, is the preliminary calculation of the forces; a comparison of the obtained values with the automated finite element calculation; insertion of the directional milling force into the deflection model; obtaining the deflections amplitude through considering the milling tool as a driven harmonic oscillator.

\subsection{Deflection MODEL}

During the processing of the thin-walled element by the end-mill, undesirable deviations occur in the direction of the force of the cutter pressure on the surface. An additional complexity of calculating deflections is the variable distribution of stiffness in each section. It is caused by the geometry of a thin-walled element, similar to an impeller blade. The considered model with a larger thickness at the base and tapering towards the end is presented in Fig 1. The model is shear, undeformable and represents the static force interaction. It is used for a further rough deflections value validation.

The basis for the equation of the deflection model is the Castigliano's second theorem, which states that the displacement $\Delta$ of the point of application of the generalized force is equal to the partial derivative of the complementary strain energy $U_{c}$ with respect to this generalized force $F$ :

$$
\Delta=\frac{\partial U_{c}}{\partial F}
$$

The complementary strain energy of bending:

$$
U_{c}=\int_{0}^{L} \frac{M^{2}}{2 E I} d x
$$

where Young's modulus $E$ is a measure of the material stiffness [25]. The bending moment $M$ and moment of inertia $I$ are functions of $x$ :

$$
M=F \cdot x
$$

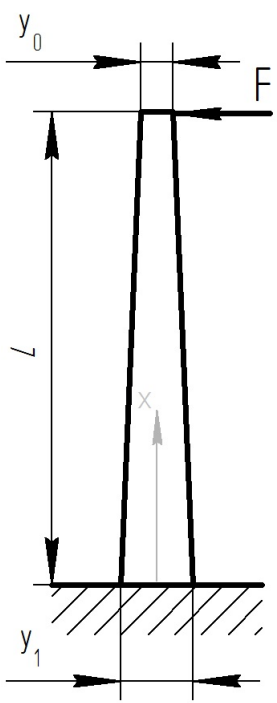

Figure 1. Model of a sample under the directional force.

$$
I=\frac{b \cdot\left(y_{0}+\alpha x\right)^{3}}{12}
$$

where $b$ is a beam width.

The increasing thickness measure $\alpha$ is represented as $\left(y_{1}-y_{0}\right) / L$. Combining the expressions into the complementary strain energy of bending (2):

$$
U_{c}=\frac{6 \cdot F^{2}}{E b} \int_{0}^{L} \frac{x^{2}}{\left(y_{0}+\alpha x\right)^{3}} d x
$$

Combining the expressions into the Castigliano's equation (1), the deflection:

$$
\Delta=\frac{\partial U_{c}}{\partial F}=\frac{12 \cdot F}{E b} \int_{0}^{L} \frac{x^{2}}{\left(y_{0}+\alpha x\right)^{3}} d x
$$

As a result of calculations, where a Young's modulus for aluminium $E=69 \mathrm{GPa}, y_{1}=9.75 \mathrm{~mm}, y_{0}=4.75$ $\mathrm{mm}, b=40 \mathrm{~mm}, L=70 \mathrm{~mm}, F=184 \mathrm{~N}$ - obtained from the section 3.2 , the deflection $\Delta=0.1642 \mathrm{~mm}$.

\subsection{Directional CUtTing ForCe MODEL}

To determine the forces acting in the cross section of the sample, as the most influencing factors of undesirable deflections, it is necessary to define the components of the cutting forces Fig. 2

The scheme of the cutting forces during the milling depends on the machining method and the type of the cutter. For the processing of thin-walled parts, such profiles of blades, end mills are used. The finishing climb milling of an aluminium alloy sample is presented. Climb milling provides a surface with a lower roughness and a higher accuracy. The tooth cuts into the material at a point A Fig. 2, causing a number of forces directed towards the surface. But, in the case of thin-walled elements that are not deprived of the freedom degree from the back side, the effect of deflection by the cutter of an element leads to the processing errors. 


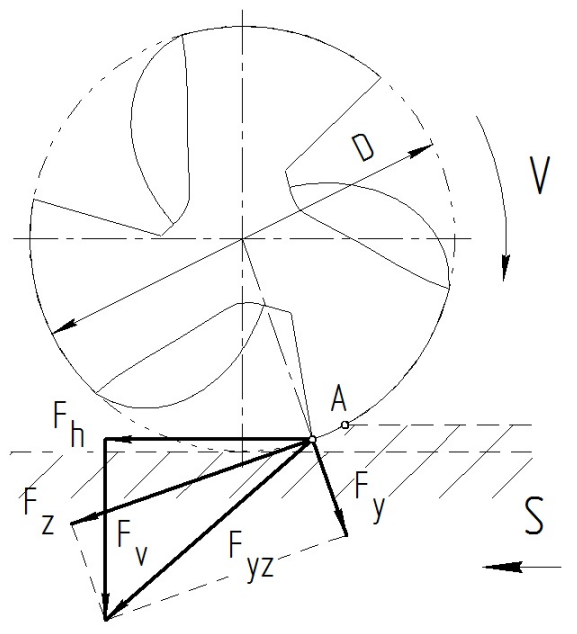

Figure 2. Components of cutting forces in the end milling.

In accordance with the reference book [26], the total tangential force $F_{z}$ and the radial force $F_{y}$ have a resultant $F_{y z}$, which can be decomposed into two forces - the lengthwise $F_{h}$ and the transverse force $F_{v}$. In the case of the processing model, the lengthwise force $F_{h}$ is directed along the line of the material removal. The force $F_{v}$ is directed perpendicularly to the force $F_{h}$, in the direction of the supposed blade sample deflection.

To determine the transverse force $F_{v}$, which is responsible for the occurrence of deviations, the total tangential force $F_{z}$ is needed:

$$
F_{z}=\frac{10 \cdot C_{p} \cdot t^{x} \cdot S_{z}^{y} \cdot B^{n} \cdot z}{D^{q} \cdot n^{w}} \cdot K_{M P}
$$

where $C_{p}$ is the coefficient that takes into account the physical and mechanical properties of the material, tabulated value; $x, y, w, q$ - exponents that depend on the type of machining, the material of the part and the material of the cutting tool; $t$ - depth of cutting, $\mathrm{mm} ; S_{z}$ - feed, $\mathrm{mm} / \mathrm{rev} ; B$ - cutting width, $\mathrm{mm} ; z$ - number of cutter teeth; $D$ - diameter of the mill, $\mathrm{mm} ; n$ - rotational speed, $\min ^{-1} ; K_{M P}$ - the general correction factor, which takes into account the quality of the processed material [26].

As a result of calculations, where a cutting depth $t=0.25 \mathrm{~mm}$, cutting width $B=3 \mathrm{~mm}$, diameter of the contact area of the end spiral three-blade mill $D$ $=7.5 \mathrm{~mm}$, and the material of the part - aluminium alloy, the value of the tangential force is $F_{z}=263.2 \mathrm{~N}$. Forces $F_{z}$ and $F_{v}$ are in the ratio [26], as $F_{z}: F_{v}=$ 0.7-0.9. Based on the ratio, the calculated value of the transverse force is $F_{v}=184 \mathrm{~N}$. The values obtained are necessary for a further comparison with the finite element analysis.

\subsection{HARMONIC RESPONSE MODEL}

In classical mechanics, a harmonic oscillator is a system that, when displaced from its equilibrium position, experiences a restoring force $F$ proportional to the displacement $x$ :

$$
F=-k \cdot x-b \cdot v
$$

where, $k$ is a positive real number, characteristic of the spring; $x$ - amount of the displacement; $b$ - is a constant that depends on the properties of the environment and the dimensions of the object; $v$ - is the velocity of the object.

Driven harmonic oscillators are damped oscillators further affected by an externally applied force $F(t)$. Let us assume a driving force is $F=F_{0} \cdot \cos \left(\omega_{\text {ext }} t\right)$ [27], then the totals force:

$$
F=F_{0} \cdot \cos \left(\omega_{\text {ext }} t\right)-k \cdot x-b \cdot v
$$

The equation of motion, $F=m \cdot a$, becomes:

$$
\frac{m \cdot d^{2} x}{d t^{2}}=F_{0} \cdot \cos \left(\omega_{e x t} t\right)-k \cdot x-\frac{b d x}{d t}
$$

After a steady state has been reached, the position varies as a function of time:

$$
x(t)=A \cdot \cos (\omega \cdot t+\varphi)
$$

where, $\omega=\omega_{\text {ext }}$ is the angular frequency of the driving force. The amplitude of oscillation:

$$
A=\frac{F_{0}}{m \cdot\left(\left(\omega_{0}^{2}-\omega^{2}\right)^{2}+\left(\frac{b \cdot \omega}{m}\right)^{2}\right)^{1 / 2}}
$$

where, $\omega_{0}^{2}=k / m$. $\omega_{0}$ is the natural frequency of the undamped oscillator. When the frequency of the driving force is close to the natural frequency and the drag force is small, then the denominator in the above expression becomes very small and the amplitude becomes very large. This increase in amplitude is called resonance, $\omega=\omega_{0}$ is resonance frequency.

Due to the geometrical complexity of the sample, a calculation for a slab of a similar cross sectional area and stiffness is performed. The size of the slab: $70 \mathrm{~mm}$ long, $40 \mathrm{~mm}$ wide, $8.25 \mathrm{~mm}$ thick, and stiffness $k=1.14 \cdot 10^{6}$. According to the section 3.1 the stiffness of the model is $k=F / \Delta=1.12 \cdot 10^{6}$. To show the response of the slab to the force in the graph, the Duhamel's integral is used:

$$
u(F, t)=\frac{1}{m \omega_{0}} \int_{0}^{t} F_{\tau} e^{-h(t-\tau)} \sin (\omega(t-\tau)) d \tau
$$

The graph on the Fig. 3 displays the dependency of the oscillation amplitude on time for the functions of the force for resonance $F_{r}(t)$ and for normal conditions $F_{n}(t)$. It is assumed that, under normal conditions, the frequency $f n=35 \mathrm{~Hz}$ corresponds to the generated oscillations during a conventional machining at a $750 \mathrm{rpm}$ by the three blade end-mill. Based on the calculation results, the amplitude of the oscillation $A_{n}=$ $0.2247 \mathrm{~mm}$, the resonance amplitude $A_{r}=4.023 \mathrm{~mm}$.

\section{Results}

Results are based on a finite element analysis. The values obtained as a result of previously manual calculated models and automated are compared. 


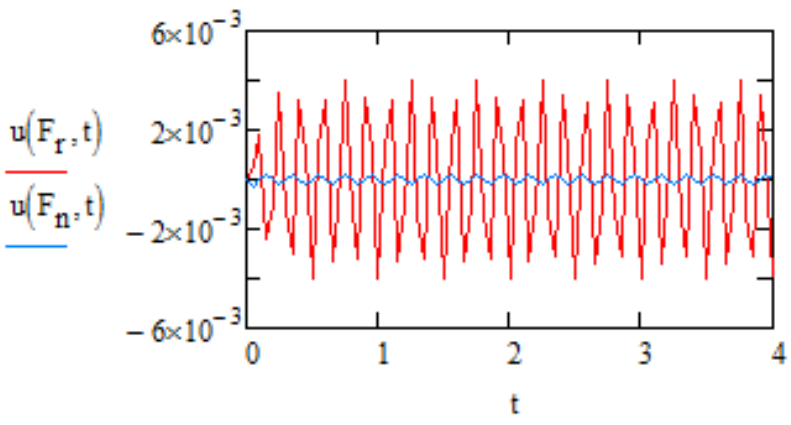

Figure 3. The amplitudes of oscillations at resonance $u\left(F_{r}, t\right)$ and normal conditions $u\left(F_{n}, t\right)$.

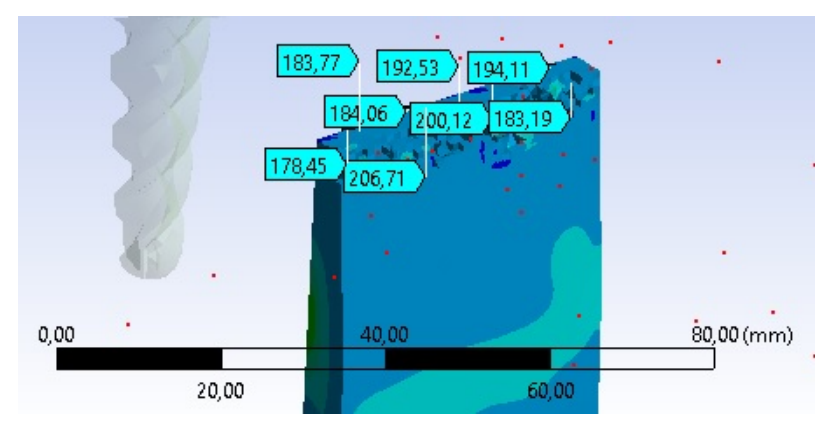

Figure 4. Model of analysis and forces probes.

\subsection{END MILLING ANALYSIS}

CAD models of a helical conical three-blade end mill and a thin-walled sample element with a variable distribution of stiffness have been designed and imported into CAE program. Based on the model defined in the section 3.1. the length $L$ of the sample is $70 \mathrm{~mm}$. Width $\mathrm{b}$ is $40 \mathrm{~mm}$. The thickest $y_{1}$ and the thinnest $y_{0}$ regions of the middle section of the part are $9.75 \mathrm{~mm}$ and $4.75 \mathrm{~mm}$ respectively. The thickness at the top edges (point 1, point 3) Fig. 5 is $3.75 \mathrm{~mm}$.

The material, contact surfaces of the sample and cutters, boundary surfaces and a mesh for the finite element calculation are set. The estimated allowance for the finishing process is in the range of up to 500 $\mu \mathrm{m}$. Therefore, the density of the mesh of the sample is much smaller than that, and equals $0.5 \mathrm{~mm}$.

The initial position of the milling cutter and the sample is calculated at the CAD design step. A particular attention should be paid to the position of the sample and the tool, both relative to each other, and relative to the coordinate system. In the CAE environment, both elements are tied to the global coordinate system. A cylindrical coordinate system is additionally assigned to the milling cutter. Having the specified parameters above, the dynamic parameters are set: axial rotation of the milling cutter and axial movement of the part on the machining length.

One of the main parameters of interest in this article are the forces acting on the thin-walled element in the transverse direction. The simulated force $F_{v \text {.fea }}$ can be obtained by taking stress probes from the back of the sample along the cutting area, as it can be seen

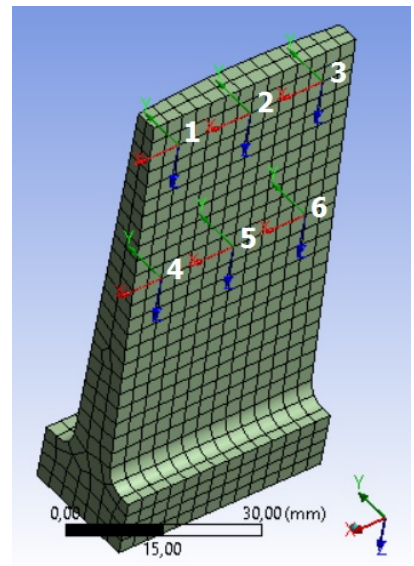

Figure 5. Set of critical points.

\begin{tabular}{cc}
\hline Critical points & Deflection values, $\mathbf{m m}$ \\
\hline 1 & 0.17984 \\
2 & 0.15186 \\
3 & 0.17984 \\
4 & 0.06180 \\
5 & 0.05319 \\
6 & 0.06180 \\
\hline
\end{tabular}

TABLE 1. Deflections estimation.

in Fig. 4. It is necessary for the comparison with the calculated force $F_{v \text {.calc }}=184 \mathrm{~N}$.

Analysing the obtained values, it can clearly be seen that the finite element analysis allows getting the approximate values of forces.

However, it is necessary to take into account the difference in values, depending on the location of the probes, despite the constant cutting parameters. That may be caused by inaccuracies in the context of this type of analysis. The error of the applied method is $12 \%$ on average.

Considering six points on the surface Fig. 5 five of them are critical - they are at the edges of the sample and far from the fixation site. Consequently, at these points, the maximum deviation from the initial state is presumably observed.

The point 5 is the test point where the lowest deflection is expected. The location of the points approximately matches with the lines of the end milling.

By loading the critical points by forces in sequence, it is possible to estimate the magnitude of the deviations in each region. The direction of the forces is set along the $Y$ axis of the local coordinate systems. The $Y$ axis is perpendicular to the sample surface. The values of the deviations picked at different levels are different; it is caused by variable a distribution of stiffness of the sample [28, 29].

The values obtained by the analysis allow to clearly evaluate the degree of the maximum deflection values of the sample in different regions Fig. 6. The results of the analysis are listed in Tab. 1. The maximum 


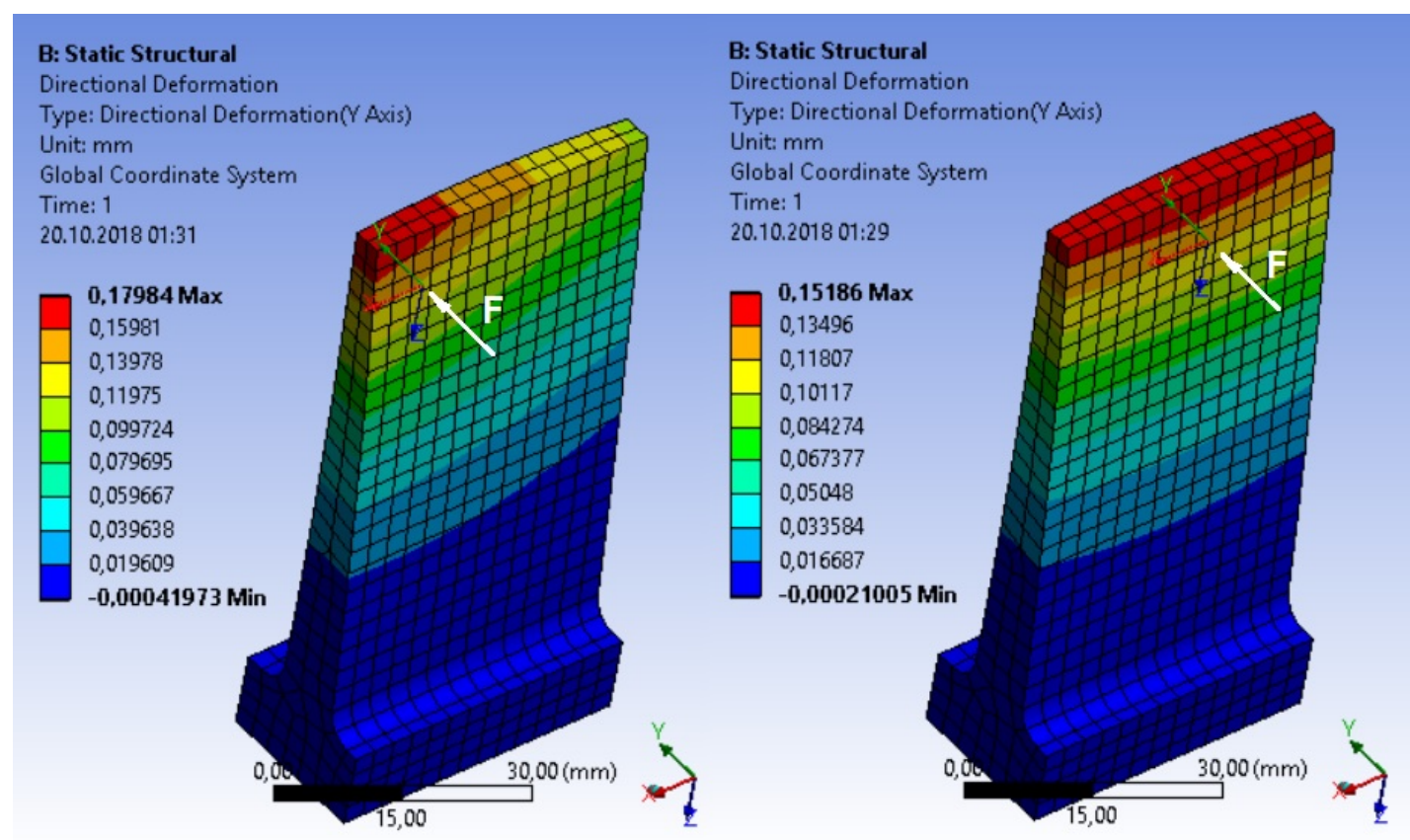

Figure 6 . Sample deflection at point 1 and 2 by $0.17984 \mathrm{~mm}$ and $0.15186 \mathrm{~mm}$ respectively.

deflection value for the sample thickness $3.75 \mathrm{~mm}$ at points 1 and 3 is $0.17984 \mathrm{~mm}$.

At the section 3.1 the deflection $\Delta=0.1642 \mathrm{~mm}$ is obtained. That simplified model geometrically represents the force interaction at the level of the second point of the sample where the thickness is $4.75 \mathrm{~mm}$. The deflection at this point according to the FE model is $0.15186 \mathrm{~mm}$, which is quite similar. The different calculation methods allow to be sure that in the process of building of the FE model no errors were made. Also, the simplified model allows to roughly estimate what value is expected as a result of the FE analysis.

The FE analysis in the CAE software gives more possibilities due to working with a complex $3 \mathrm{D}$ model thus the more accurate calculations can be performed.

\subsection{MODAL ANALYSIS}

Calculating of the natural frequency of the structure should especially be analysed if there are tendencies to the occurrence of oscillations in the process of treatment. This calculation makes it possible to define the Harmonic Response oscillations further, i.e. calculate the maximum amplitude of oscillation.

The interaction of a rotating cutter with a certain frequency and a thin-walled element can be represented as a mechanical system with cyclic loads. The response of a system to a dynamic loading can be among the following [30]:

(1.) the intensity of the response can converge;

(2.) the system can constantly oscillate;

(3.) the intensity of the system can diverge.

In all cases, the 3-rd type of the response should be avoided since the system could collapse.
If the excitation load has a frequency which becomes close to the natural frequency of the system, the oscillations increase, and if the frequencies coincide, a resonance phenomenon occurs with subsequent negative consequences. Therefore, it is critical to be able to calculate the natural frequency of the system. For this purpose, the finite-element analysis of frequencies and oscillations is presented.

This analysis calculates the natural frequency based on the geometry and material of the sample, and allows to determine the resonant frequency.

The modal analysis settings are: Max Modes to Find - 6 (each of them has a tendency to oscillate in different directions), Fixed Support - the base surface of the model. The result is shown in Fig. 7 the output listed in Tab. 2

The first mode $-1728.4 \mathrm{~Hz}$ is the most significant, since at this frequency, the sample is going to oscillate in the same direction as during the lengthwise processing.

\subsection{Harmonic Response Analysis}

Having a modal analysis, the Harmonic Response analysis can be performed. Any sustained cyclic load produces a sustained cyclic response (a harmonic response) in a structural system. The harmonic response analysis gives the ability to predict the sustained dynamic behaviour of structures, thus enabling to verify whether or not the designs will successfully overcome the resonance, fatigue, and other harmful effects of forced vibrations 31.

Harmonic Response analysis settings are: previously defined Modal analysis, Fixed Support - base surface of the model, analyzed frequency is in the range from 0 to $2000 \mathrm{~Hz}$, input Nodal Force load at the critical 


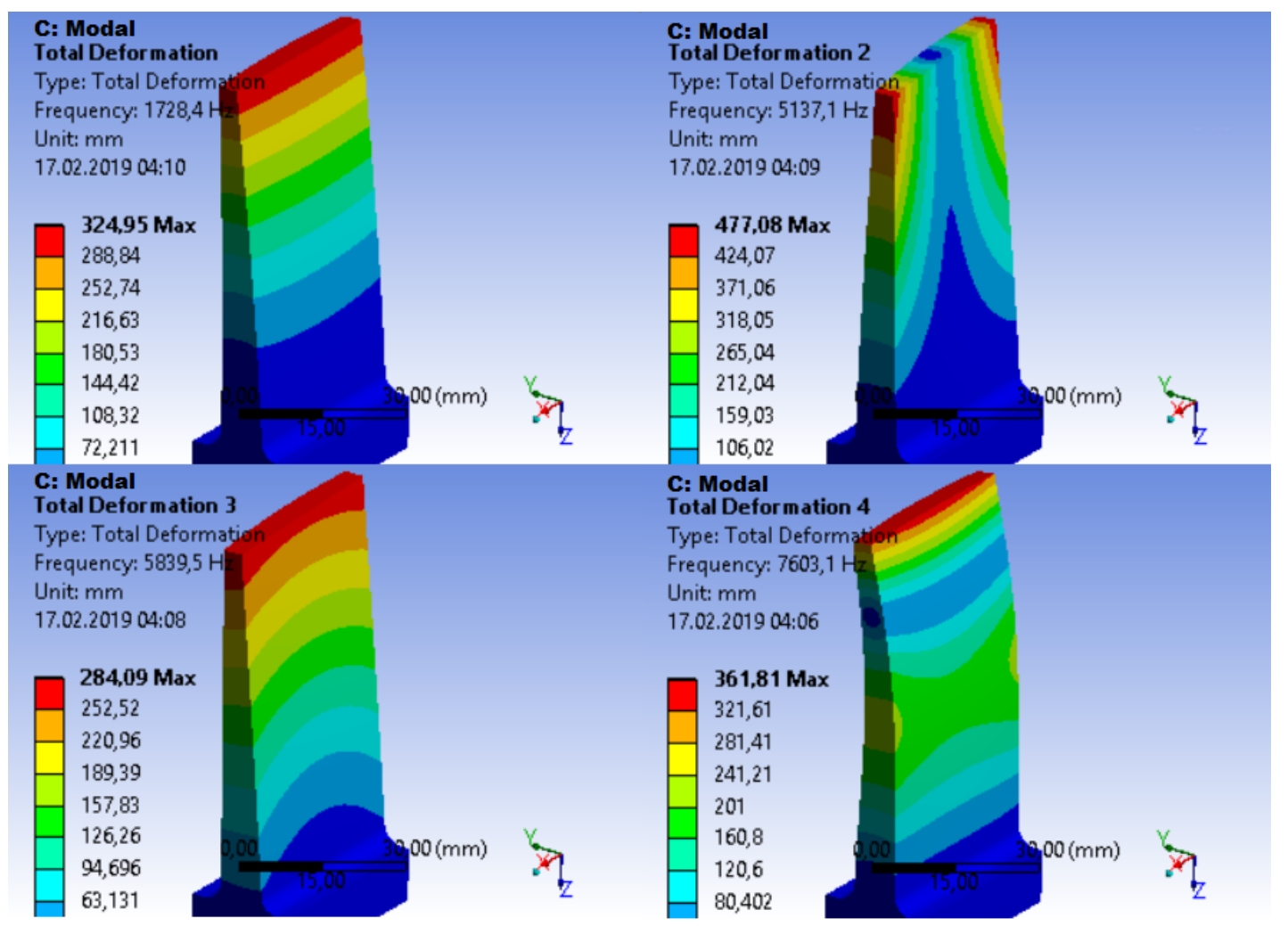

FiguRE 7. Oscillations under different natural frequencies.

\begin{tabular}{cc}
\hline Mode & Frequency, $\mathbf{H z}$ \\
\hline 1 & 1728.4 \\
2 & 5137.1 \\
3 & 5839.5 \\
4 & 7603.1 \\
5 & 13097 \\
6 & 17771 \\
\hline
\end{tabular}

TABLE 2. Natural frequency analysis output.

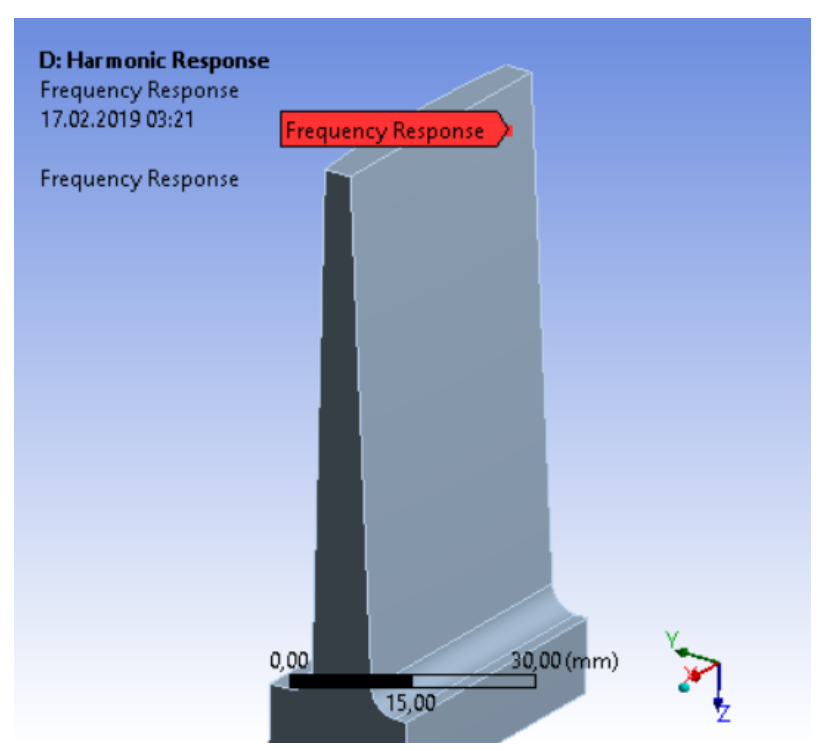

FiguRE 8. Frequency Response location. point (thin end of the sample) - 184 N. The Frequency Response is taken from the critical point 3 location Fig. 8

The graphs of Oscillation Amplitude and Phase Angle are presented on the Figure 9, values for each frequency and corresponding amplitude, phase angle are presented in the Tabl. 3

The amplitude is the maximum value of the oscillation of the sample under the specified load and corresponding frequency. The phase angle is a measure of the time by which the load lags (or leads) a frame of reference.

The range from 0 to $2000 \mathrm{~Hz}$ is significant to analyse as the natural frequency lies in this range $-1728.4 \mathrm{H}_{z}$. If the frequency of the cyclic load matches with the natural frequency, the oscillations increase and a resonance occurs.

In confirmation, from the data received Fig. 9 , Tab. 3 a large increase of the amplitude of the sample oscillations - $2.7934 \mathrm{~mm}$ at the frequency $-1720 \mathrm{~Hz}$ 

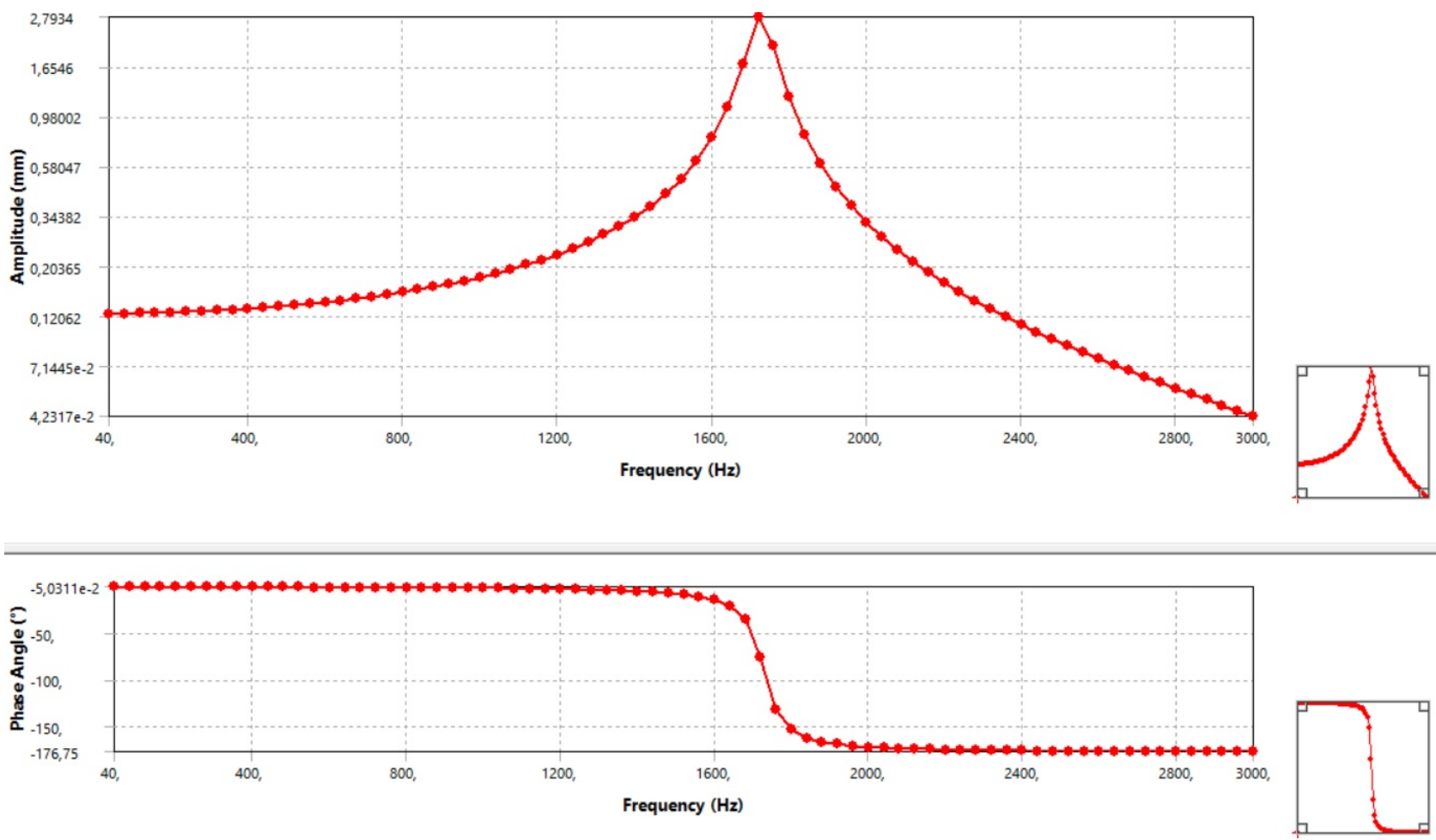

Figure 9. Oscillation Amplitude of the deflection of the sample and Phase Angle.

\begin{tabular}{ccc}
\hline Frequency, $\mathbf{H z}$ & Amplitude, mm & Phase Angle, deg. \\
\hline 40 & 0.12408 & -0.0503 \\
120 & 0.12458 & -0.1516 \\
250 & 0.12649 & -0.32113 \\
500 & 0.13455 & -0.68722 \\
750 & 0.15072 & -1.1669 \\
1000 & 0.18183 & -1.9077 \\
1250 & 0.24956 & -3.3521 \\
1500 & 0.4692 & -7.8414 \\
1720 & 2.7934 & -76.045 \\
1875 & 0.61746 & -165.95 \\
2000 & 0.32309 & -171.92 \\
\hline
\end{tabular}

TABlE 3. Shortened list of Frequencies and corresponding Amplitudes and Phase Angles.

could be noticed. It shows that the system starts to resonate with the frequency of the input loading.

The expected rotation of the milling tool for a conventional machining is near $750 \mathrm{rpm}$. It corresponds to the frequency $12.5 \mathrm{~Hz}$. The milling tool has three blades, so expected frequency of the contact of the blades with the surface is tripled. Thus the frequency of interest is near $37.5 \mathrm{~Hz}$, which is lower than the natural frequency, so milling processing can be safely performed. Finally, according to the Table 3, the maximum oscillation amplitude of the sample at $40 \mathrm{~Hz}$ is $0.12408 \mathrm{~mm}$. The same way, during the high speed machining at $15000 \mathrm{rpm}$ the frequency of interest is $750 \mathrm{~Hz}$, and the deflection is 0.15072 respectively.

As a result of the calculations in the section 3.3 the osculation amplitude for the conventional machining is $0.2247 \mathrm{~mm}$, whereas according to the FE analysis, it is $0.12408 \mathrm{~mm}$ for the conventional machining and $0.15072 \mathrm{~mm}$ for a high-speed one. The difference is expected since, unlike the simplified model, the FE model has a thick base thus it has a higher natural frequency so it is more stable to oscillations.

The difference in results shows the disadvantage of the simplified model calculation in the section 3.3 in contrast with the finite element simulation. The complexity of the manual mathematical description of the geometry imposes restrictions on the accuracy of calculations for the specific model.

\section{Discussion}

In terms of processing of thin-walled parts, Harmonic Response analysis brings the perspective to estimate deflections at different frequencies. Modern highspeed machining equipment allows the flexibility to 
choose the most efficient processing speed. Negative factors, such as large deviations or even resonance, can be avoided by increasing or decreasing the number of tool's teeth and revolutions of the cutting tool.

\section{Conclusion}

The static and dynamic methods of loading of the thinwalled sample with variable low stiffness are presented. A deflection evaluation is performed. An analysis of a sample is made, as a result, the cutting force components are defined; the relative transverse force $F_{v \text {.calc }}$ is $184 \mathrm{~N}$, which is similar to $F_{v \text {.fea }}$. The error of the applied method is $12 \%$ on average.

The modelling of the deflections is implemented. As a result of a static analysis, the maximum deviation at the thinnest region of the part is $0.17984 \mathrm{~mm}$. As a result of the frequency analysis, the maximum oscillation amplitude of the sample at a normal processing frequency $40 \mathrm{~Hz}$ is $0.12408 \mathrm{~mm}$ and at a high-speed one $-750 \mathrm{~Hz}$, it is $0.15072 \mathrm{~mm}$. In a further research, the presented methods are considered for creating processing programs that take into account the geometry of the part and compensate most of the undesirable deflections.

\section{REFERENCES}

[1] L. Breńkacz, G. Zywica, M. Bogulicz. Analysis of dynamical properties of a $700 \mathrm{kw}$ turbine rotor designed to operate in an orc installation. Diagnostyka pp. 17-23, 2016.

[2] A. Gebura, M. Stefaniuk. Monitoring the helicopter transmission using the FAM-C diagnostic method. Diagnostyka 18:75-85, 2017.

[3] E. Pásztor, B. Varga. Energy and aerodynamic examination of slightly backward leaning impeller blading of small centrifugal compressors. Periodica Polytechnica Transportation Engineering 43:199-205, 2015. DOI:10.3311/PPtr.8093.

[4] F. Song, Y. Ni, Z. Tan. Optimization design, modeling and dynamic analysis for composite wind turbine blade. Procedia Engineering 16:369-375, 2011. DOI:10.1016/j.proeng.2011.08.1097

[5] M. Amine Chelabi, M. Kamel Hamidou, M. Hamel. Effects of cone angle and inlet blade angle on mixed inflow turbine performances. Periodica Polytechnica Mechanical Engineering 61:225-233, 2017. DOI:10.3311/PPme.9890.

[6] M. Pompa. Computer aided process planning for high-speed milling of thin-walled parts: strategy-based support. Ph.D. thesis, University of Twente, The Netherlands, 2010. DOI:10.3990/1.9789036530408

[7] V. Ivanov. Process-oriented approach to fixture design. In Design, Simulation, Manufacturing: The Innovation Exchange, pp. 42-50. Springer, 2018. DOI:10.1007/978-3-319-93587-4_5.

[8] S. Dobrotvorskiy, Y. Basova, M. Ivanova, et al. Forecasting of the productivity of parts machining by high-speed milling with the method of half-overlap. Diagnostyka 19:37-42, 2018. DOI:10.29354/diag/93136
[9] A. Permyakov, S. Dobrotvorskiy, L. Dobrovolska, et al. Computer modeling application for predicting of the passing of the high-speed milling machining hardened steel. In Design, Simulation, Manufacturing: The Innovation Exchange, pp. 135-145. Springer, 2018. DOI:10.1007/978-3-319-93587-4_15.

[10] V. Karpus, V. Ivanov. Choice of the optimal configuration of modular reusable fixtures. Russian Engineering Research 32:213-219, 2012. DOI:10.3103/S1068798X12030124

[11] V. Ivanov, I. Dehtiarov, Y. Denysenko, et al. Experimental diagnostic research of fixture. Diagnostyka 19:3-9, 2018. DOI:10.29354/diag/92293

[12] C. Eksioglu, Z. Kilic, Y. Altintas. Discrete-time prediction of chatter stability, cutting forces, and surface location errors in flexible milling systems. Journal of Manufacturing Science and Engineering 134:061006, 2012. DOI:10.1115/1.4007622.

[13] G. Bolar, A. Das, S. N. Joshi. Measurement and analysis of cutting force and product surface quality during end-milling of thin-wall components.

Measurement 121:190-204, 2018. DOI:10.1016/j.measurement.2018.02.015

[14] T. Umezu, D. Kono, A. Matsubara. Evaluation of on-machine measuring method for dynamic stiffness of thin-walled workpieces. Procedia CIRP 77:34-37, 2018. DOI:10.1016/j.procir.2018.08.204

[15] A. Procházka, J. Uhlír, P. W. J. Rayner, N. G. Kingsbury (eds.). Signal Analysis and Prediction. Applied and Numerical Harmonic Analysis, chap. System Identification, pp. 163-173. Birkhäuser, Boston, MA, 1998 .

[16] C. Pappalardo, D. Guida. Development of a new inertial-based vibration absorber for the active vibration control of flexible structures. Engineering Letters 26:372-385, 2018. Accessed: 02 February 2019.

[17] C. Pappalardo, D. Guida. System identification and experimental modal analysis of a frame structure. Engineering Letters 26:56-68, 2018.

[18] J.-N. Juang, M. Q. Phan. Identification and Control of Mechanical Systems. Cambridge University Press, 2001. DOI:10.1017/CBO9780511547119

[19] Y. Altintas, O. Tuysuz, M. Habibi, Z. Li. Virtual compensation of deflection errors in ball end milling of flexible blades. CIRP Annals 67(1):365-368, 2018. DOI:10.1016/j.cirp.2018.03.001.

[20] V. F. Mozgovoy, K. B. Balushok, I. I. Kotov, B. M. K. Strategies for processing blades on CNC machining centers with variable $3 \mathrm{D}$ correction. Aerospace Engineering and Technology 7:22-28, 2013.

[21] V. Schulze, P. Arrazola, F. Zanger, J. Osterried. Simulation of distortion due to machining of thin-walled components. Procedia CIRP 8:45-50, 2013. DOI:10.1016/j.procir.2013.06.063

[22] Dr. Drang. An application of Castigliano's Second Theorem with Octave. https://leancrew.com/all-this/2009/10/ an-application-ofcastilianos-second-theorem-with-octave/, 2009. Accessed: 01 June 2018. 
[23] Dr. Drang. Revisiting Castigliano with SciPy. www.leancrew.com/all-this/2013/01/ revisiting-castigliano-with-scipy/, 2013. Accessed: 02 June 2018.

[24] S. Dobrotvorskiy, Y. Basova, S. Kononenko. Improvment of technology of milling parts with uneven stiffness. Collection of scientific works: Open information and computer integrated technologies 72:105-111, 2016.

[25] K. Grote, E. K. Antonsson (eds.). Springer Handbook of Mechanical Engineering. Springer-Verlag Berlin Heidelberg, 2009.

[26] A. Kosilova, R. Meshcheryakova (eds.). Reference book of a technologist-machine engineer. Springer-Verlag Berlin Heidelberg, Moscow, 4th edn., 1985.

[27] The University of Tennessee, Department of Physics and Astronomy. Damped and driven oscillations . http://labman.phys.utk.edu/phys221core/modules/ m11/damped_and_driven_oscillations.html Accessed: 02 October 2018.
[28] Sonnerlind, H. Modeling linear elastic materials How difficult can it be? https://comsol.com/blogs/ modeling-linear-elastic-materialshow-difficult-can-it-be/. Accessed: 03 June 2018.

[29] Datta, S. Computing stiffness of linear elastic structures: Part 1.

https://comsol.com/blogs/computing-

stiffness-linear-elastic-structures-part-1/ Accessed: 07 June 2018.

[30] Cyprien. What is FEA modal analysis? https://thewikihow.com/video_-_fdVg1-9yI Accessed: 27 September 2018.

[31] Chapter 4: Harmonic response analysis. www.ansys. stuba.sk/html/guide_55/g-str/GSTR4.htm. Accessed: 03 October 2018 . 